\title{
Effect of targeted disruption of STAT4 and STAT6 on the induction of experimental autoimmune encephalomyelitis
}

\author{
Tanuja Chitnis, ${ }^{1}$ Nader Najafian, ${ }^{2}$ Christina Benou, ${ }^{1}$ Alan D. Salama, ${ }^{2}$ Michael J. Grusby, ${ }^{3}$ \\ Mohamed H. Sayegh, ${ }^{2}$ and Samia J. Khoury ${ }^{1}$ \\ ${ }^{1}$ Center for Neurologic Diseases, and \\ ${ }^{2}$ Laboratory of Immunogenetics and Transplantation, Brigham and Women's Hospital, Harvard Medical School, Boston, \\ Massachusetts, USA \\ ${ }^{3}$ Department of Immunology and Infectious Diseases, Harvard School of Public Health, Boston, Massachusetts, USA \\ Address correspondence to: Samia J. Khoury, 77 Avenue Louis Pasteur, Room 714, Center for Neurologic Diseases, \\ Brigham and Women's Hospital, Boston, Massachusetts 02115, USA. \\ Phone: (617) 525-5370; Fax: (617) 525-5252; E-mail: skhoury@rics.bwh.harvard.edu.
}

Received for publication February 20, 2001, and accepted in revised form July 16, 2001.

\begin{abstract}
Experimental autoimmune encephalomyelitis (EAE) is mediated by myelin-specific $\mathrm{CD}^{+} \mathrm{T}$ cells secreting Th1 cytokines, while recovery from disease is associated with expression of Th2 cytokines. Investigations into the role of individual cytokines in disease induction have yielded contradictory results. Here we used animals with targeted deletion of the STAT4 or STAT6 genes to determine the role of these signaling molecules in EAE. The STAT4 pathway controls the differentiation of cells into a Th1 phenotype, while the STAT6 pathway controls the differentiation of cells into a Th2 phenotype. We found that mice deficient in STAT4 are resistant to the induction of EAE, with minimal inflammatory infiltrates in the central nervous system. In contrast, STAT6-deficient mice, which have a predominantly Th1 phenotype, experience a more severe clinical course of EAE as compared with wild-type or STAT4 knockout mice. In addition, adoptive transfer studies confirm the regulatory functions of a Th2 environment in vivo. These novel data indicate that STAT4 and STAT6 genes play a critical role in regulating the autoimmune response in EAE.
\end{abstract}

J. Clin. Invest. 108:739-747 (2001). DOI:10.1172/JCI200112563.

\section{Introduction}

Experimental autoimmune encephalomyelitis (EAE) is a T cell-mediated disease that is used as a model for the study of multiple sclerosis. In EAE, cells expressing Th1 cytokines predominate and mediate inflammatory damage $(1,2)$, while cells expressing Th2 cytokines have been associated with remissions and recovery from disease (3-5). However, the effect of targeted deletion of individual cytokines on EAE has led to unexpected results. IL- $4^{-/-}$mice were reported by some investigators to develop disease similar to wild-type mice $(6,7)$, while other investigators reported a more severe disease in these mice (8). Transgenic expression of IL-4 in $\mathrm{T}$ cells does not protect from EAE (7). IFN- $\gamma^{-/}$and IFN- $\gamma$ receptor ${ }^{-/}$mice develop severe disease $(9,10)$, and treatment of mice with anti-IFN- $\gamma$ Ab worsened disease $(11,12)$. These apparently conflicting results may be due to the redundancy of cytokine functions and the fact that cytokines may have dual roles, proinflammatory or regulatory, during the course of an immune response (13-15). Here, we investigate the role of Th1 and Th2 cytokines in EAE using mice deficient for genes that play a pivotal role in development of Th1 or Th2 immune responses. We may thereby identify possible genetic sites for future therapeutic interventions in $T$ cell-mediated autoimmune diseases.

STAT proteins are a recently identified class of molecules that mediate many cytokine-induced respons- es. These molecules are cytoplasmic proteins that are activated following phosphorylation via the Janus kinase (JAK) family of tyrosine kinases, which in turn are activated by interaction of a cytokine and its receptor. STAT proteins then dimerize, translocate to the nucleus, and bind to DNA sequences, thus regulating gene transcription. STAT6 is activated following the interaction between IL-4 and the IL-4 receptor on the surface of cells and is critical for the development of Th2 cells $(16,17)$. Mice deficient in STAT6 display a reduction in Th2 cytokine production, decreased IL-4-induced B cell proliferation, and reduced $\operatorname{IgE}(16,17)$. In contrast, STAT4 plays a pivotal role in Th1 immune responses. STAT4 is activated after IL-12 interacts with the IL-12 receptor, inducing transcription of IFN- $\gamma(18)$. Mice deficient in STAT4 lack IL-12-induced IFN- $\gamma$ production and Th1 differentiation $(19,20)$ and display a predominant Th2 phenotype (20). Further studies showed that STAT4/STAT6 double knockout mice are able to mount a Th1 response, indicating that there is an alternate pathway for Th1 differentiation that is suppressed by the presence of Th2 cytokines as in STAT6 ${ }^{-/-}$mice (21). STAT4 $4^{-/-}$mice have been recently reported to be resistant to lymphocytic choriomeningitis virus-induced diabetes (22), but reject a fully allogeneic transplanted organ at the same tempo as STAT6 ${ }^{-/-}$and wild-type mice (23). 
In this report, we used STAT4- and STAT6-deficient mice to investigate the regulatory functions of Th1 and Th2 cells in EAE induced by myelin oligodendrocyte glycoprotein (MOG).

\section{Methods}

Mice. STAT4 $4^{-/-}$and STAT6 $6^{-/-}$mice were generated by M.J. Grusby as described previously $(17,20)$ and backcrossed onto a C57BL $/ 6$ background for at least ten generations. C57BL $/ 6$ wild-type and TCR- $\alpha \beta^{-/-}$mice on a C57BL/ 6 background were purchased from The Jackson Laboratories (Bar Harbor, Maine, USA). The maintenance of the facility and use of animals is in full compliance with the Laboratory Animal Welfare Act and the Health Research Extensions Act. Female mice at 6-8 weeks of age were used for experiments.

EAE induction with MOG. MOG peptide 35-55 (MOG 35-55) (M-E-V-G-W-Y-R-S-P-F-S-R-O-V-H-L-Y-R-N-G-K) corresponding to the mouse sequence was synthesized by Quality Controlled Biochemicals Inc. Division of BioSource International (Hopkinton, Massachusetts, USA) and purified to greater than $99 \%$ by HPLC. C57BL/ 6 wild-type, STAT4-deficient, and STAT6-deficient mice (3-8 mice per group) were immunized subcutaneously in the flanks with 150-200 $\mu \mathrm{g}$ of MOG peptide in $0.1 \mathrm{ml} \mathrm{PBS}$ and $0.1 \mathrm{ml}$ CFA containing $0.4 \mathrm{mg}$ Mycobacterium tuberculosis (H37Ra; Difco Laboratories, Detroit, Michigan, USA) and injected intraperitoneally with $200 \mathrm{ng}$ pertussis toxin (List Biological Laboratories Inc., Campbell, California, USA) on the day of immunization and 2 days later. EAE was scored as described previously (24): grade 1, limp tail or isolated weakness of gait without limp tail; grade 2, partial hind leg paralysis; grade 3 , total hind leg or partial hind and front leg paralysis; grade 4, total hind leg and partial front leg paralysis; grade 5, moribund or dead animal.

Adoptive transfer. For the adoptive transfer studies, primed splenocytes are injected intraperitoneally in naive recipients that are then immunized. We elected to immunize the animals rather than reactivate the primed cells in vitro in order to allow the transferred $\mathrm{T}$ cells to be activated in vivo in the environment that we wanted to test. This model allows us to examine what happens to primed $T$ cells when they are reactivated in vivo under various conditions (Th1 versus Th2 cytokine environment). To determine the optimal number of splenocytes to be transferred, wild-type primed splenocytes were harvested on day 12 postimmunization and 25,50 , or $100 \times 10^{6}$ splenocytes were transferred into TCR- $\alpha \beta^{-/-}$ recipients. We determined that $50 \times 10^{6}$ cells induced adequate disease. That number of cells suspended in a volume of $1 \mathrm{ml}$ of PBS was used in experiments using TCR- $\alpha \beta^{-/-}$or STAT4 $4^{-/}$recipients. One day later, the recipient mice were immunized with MOG 35-55 as described above in order to activate the lymphocytes in vivo. The mice were given one dose of pertussis intraperitoneally on day 0 .

Immunization and cell culture. For in vitro experiments, mice were immunized subcutaneously in one hind foot-

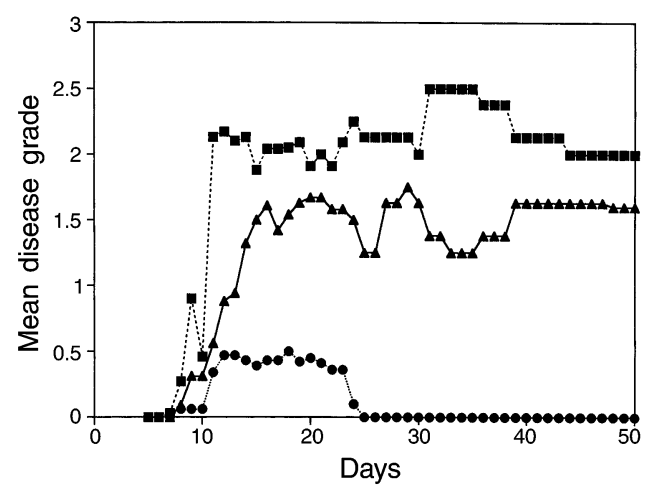

Figure 1

EAE disease course in STAT4-/-, STAT6-/-, and wild-type mice. A representative experiment showing disease induction in wild-type (triangles), STAT4 ${ }^{-/-}$(circles), and STAT6 ${ }^{-/-}$(squares) mice. Mice were immunized with MOG 35-55 and graded for disease daily. The mean daily disease grade for each group ( $n=5$ mice per group) is shown.

pad and in both flanks with an emulsion of $100 \mu$ of CFA and $100 \mu \mathrm{l}$ PBS containing $100 \mu \mathrm{g}$ of MOG 35-55. A single cell suspension was prepared from the inguinal and the draining popliteal lymph nodes or spleens. Cells were cultured in 96-well plates (Corning Costar Corp., Cambridge, Massachusetts, USA). Media used for proliferation and cytokine assays consisted of serum-free Ex Vivo 20 medium (BioWhittaker Inc., Walkersville, Maryland, USA) containing $75 \mathrm{mM} / \mathrm{ml} \mathrm{L}$-glutamine, $100 \mathrm{U} / \mathrm{ml}$ penicillin and streptomycin, $1 \mathrm{ml} / 100 \mathrm{ml}$ of media of a $100 \times$ concentrated nonessential amino acid solution, $0.1 \mathrm{mM} / \mathrm{ml}$ HEPES, $1 \mathrm{mM} / \mathrm{ml}$ sodium pyruvate (all BioWhittaker Inc.) and $0.05 \mathrm{mM} / \mathrm{ml} 2$-mercaptoethanol (Sigma Chemical Co., St. Louis, Missouri, USA). Cells were incubated at $37^{\circ} \mathrm{C}$ in humidified air containing $7 \% \mathrm{CO}_{2}$.

Proliferation assay. For proliferation assays, cells were cultured at $2 \times 10^{6}$ cells $/ \mathrm{ml}$ and $200 \mu \mathrm{l} /$ well was plated with $0,1,10$, and $100 \mu \mathrm{g} / \mathrm{ml}$ of antigen. After 48 hours of culture, $1 \mu \mathrm{Ci}^{3} \mathrm{H}$-thymidine (NEN Life Science Products Inc., Boston, Massachusetts, USA) was added in 10 $\mu \mathrm{l}$ of media to each well for another 16 hours. Cells were harvested on filter mats, dried, and counted.

Cytokine ELISA. For cytokine assays, cells were cultured at $4 \times 10^{6} \mathrm{cells} / \mathrm{ml}$ in $200 \mu \mathrm{l}$ media at antigen concentrations of $0,1,10$, and $100 \mu \mathrm{g} / \mathrm{ml}$. Supernatants for IL- 5 and IFN- $\gamma$ ELISA were collected after 48 hours of culture. Quantitative ELISAs for IL-5 and IFN- $\gamma$ were performed using paired $\mathrm{Ab}$ 's and recombinant cytokines from PharMingen (San Diego, California, USA), according to manufacturer's recommendations.

Enzyme-linked immunosorbent spot assay. Cells $\left(4 \times 10^{6}\right.$ per well) were incubated with antigen in U-bottomed plates for 24 hours. The cells were resuspended and serially diluted from a concentration of $4 \times 10^{6} / \mathrm{ml}$ down to $3 \times 10^{4} / \mathrm{ml}$, then added to nitrocellulose plates (Millipore Corp., Bedford, Massachusetts, USA). The plates were coated with $50 \mu \mathrm{l}$ primary IFN- $\gamma \mathrm{Ab}$ (clone R4-6A2; Endogen Inc., Woburn, Massachusetts, USA), 
Table 1

Disease incidence, mean maximal grade, and day of onset in STAT4 or STAT6 knockout compared with wild-type

\begin{tabular}{lccc}
\hline & Incidence & Mean maximal grade $\pm \mathrm{SE}$ & Onset $^{\mathrm{A}} \pm \mathrm{SE}$ \\
Wild-type & $16 / 16$ & $1.88 \pm 0.24$ & $10.2 \pm 0.5$ \\
STAT4 knockout & $7 / 16^{\mathrm{B}}$ & $0.46 \pm 0.2^{\mathrm{C}}$ & $10.9 \pm 0.5^{\mathrm{D}}$ \\
STAT6 knockout & $15 / 15$ & $2.7 \pm 0.1^{\mathrm{E}}$ & $8.6 \pm 0.25^{\mathrm{F}}$
\end{tabular}

${ }^{A}$ Onset is calculated only for the animals that did develop EAE. ${ }^{B} P=0.0008$ compared with wild-type and STAT $6^{-/-}$by Fisher's exact test. ${ }^{C} P=0.0002$ compared with wild-type and $P<0.0001$ compared with STAT6 ${ }^{-/-}$by Mann-Whitney test. DNot significant compared with wild-type by Mann-Whitney test. ${ }^{E} P=0.0015$ compared with wild-type by Mann-Whitney test. $F P=0.018$ compared with wildtype and $P=0.005$ compared with STAT4 $4^{-/-}$by Mann-Whitney test.

or primary IL-5 Ab (clone TRFK5; PharMingen), or primary IL-4 Ab (clone 11B11; PharMingen) at a concentration of $5 \mu \mathrm{g} / \mathrm{ml}$. One hundred microliters of the appropriate concentrations of antigen or mitogen were added to the nitrocellulose enzyme-linked immunosorbent spot (ELISPOT) assay plates. The cells were incubated for 18 hours at $37^{\circ} \mathrm{C}$, washed three times, and 50 $\mu \mathrm{l} /$ well of biotinylated IFN- $\gamma$ secondary $\mathrm{Ab}$ (clone XMG1.2; Endogen Inc.) or biotinylated IL-5 secondary $\mathrm{Ab}$ (clone TRFK4), or biotinylated IL-4 secondary $\mathrm{Ab}$ (clone BVD6-24G2) was added to the plates at a concentration of $2 \mu \mathrm{g} / \mathrm{ml}$ diluted in 1\% BSA/PBS for 5 hours at room temperature. After washing, $50 \mu \mathrm{l}$ of alkaline phosphatase (Sigma E-2636) was added to the plates at a dilution of 1:1,000 in $0.05 \%$ Tween/PBS for 2 hours at room temperature. Plates were washed twice with wash buffer and then twice in PBS. Fifty microliters of BCIP/NBT (Sigma FASTR B-5655) solution was added for 5 to 20 minutes until blue spots developed. Plates were washed with distilled water. Spots were counted using a Zeiss Stemi 100 dissecting microscope (Carl Zeiss Inc., Thornwood, New York, USA).

\footnotetext{
Figure 2

Quantitation of inflammatory cell infiltrates in the CNS of wild-type (WT), STAT4-/- (STAT4KO), and STAT6-/- (STAT6KO) mice. Quantitation of immunohistochemically stained spinal cord sections for CD4 and CD8 T cells and macrophages from C57BL/ 6 wild-type mice, STAT4 ${ }^{-/-}$mice, and STAT6 ${ }^{-/-}$mice. Spinal cord sections from three mice from each group were harvested on day 16 (a) and day 50 (b) and stained for CD4 (white bars), CD8 (dark gray bars), macrophages (black bars), and granulocytes (light gray bars). At least three sections from different levels of the cord were evaluated. Number of cells staining positive for the given marker were counted in 10 high-power fields $(\times 40)$ per section. The results for one section were totaled, and the results between sections were averaged. Error bars represent standard deviation (SD). Statistical analysis was done using the Student's $t$ test. Results from a, day 16 , show sections from STAT4 $4^{-/}$mice displayed significantly less $\mathrm{CD}^{+}(P=0.05)$ and $\mathrm{CD}^{+}$cells $(P=0.0005)$, macrophages $(P=0.02)$, and granulocytes $(P=0.004)$ than did wildtype mice. Sections from STAT6 ${ }^{-/}$mice consistently displayed more inflammatory cells than wild-type mice (however the $P$ value was not significant), and significantly more than STAT4 $4^{-/}$mice $(P<0.05)$. Results from $\mathbf{b}$, day 50 , show significantly lower numbers of $C D 4^{+}$cells in the STAT4 ${ }^{--}$group compared with wild-type $(P=0.0024)$. There were no significant differences in inflammatory infiltrates between the STAT6 $^{-/-}$and wild-type mice at day 50.
}

Immunohistology and histopathology. Spinal cords and brains were collected on day $17-21$ and at day 50 post immunization from two to four mice in each experimental group. Spinal cord tissues were embedded in OCT, quick frozen in liquid nitrogen, and kept at $-70^{\circ}$ $\mathrm{C}$ until sectioning. Cryostat sections $(10 \mu \mathrm{m})$ of spinal cords were fixed with acetone or $4 \%$ paraformaldehyde and then labeled with the $\mathrm{Ab}$ of interest: anti-mouse CD4 (clone H129.19), anti-mouse CD8a (clone 53-6.7), anti-mouse Ly-6G (clone RB6-8C5), all from PharMingen, and anti-F4/80 (clone CI:A3-1; Caltag Laboratories Inc., Burlingame, California, USA). The sections were stained using the avidin-biotin technique (Vectastain Elite kit; Vector Laboratories, Burlingame, California, USA), visualized with diamino-benzidine (Vector Laboratories), and counterstained in hematoxylin. Isotypematched Ig and omission of the primary Ab served as negative controls. Each specimen was evaluated at a minimum of three different levels of sectioning. The whole tissue section (a longitudinal spinal cord section) was evaluated for a given cellular marker at $\times 40$ magnification. Number of cells staining positive for the given marker were counted in ten $\times 40$ (high-power fields) fields per section. The results for one section were totaled, and the results between sections were averaged.

Anti-MOG $A b$ assay. Serum samples were obtained from blood of immunized or naive mice and stored at $-20^{\circ} \mathrm{C}$. MOG $35-55$ was dissolved at $1 \mu \mathrm{g} / \mathrm{ml}$ in $\mathrm{NaHCO}_{3}$ buffer $0.1 \mathrm{M}, \mathrm{pH}$ 9.6. The peptide was coated on a 96-well Nunc-Immuno Plate (Nalge Nunc International, Rochester, New York, USA) at $50 \mu \mathrm{g} /$ well for 3 hours at $37^{\circ} \mathrm{C}$. The plates were blocked overnight with $2 \% \mathrm{BSA}(\mathrm{wt} / \mathrm{vol})$ in PBS at $4^{\circ} \mathrm{C}$. The serum samples (diluted 1:50 in PBS) were added, then serially diluted on the ELISA plate. The plates were incubated for 3 hours at $37^{\circ} \mathrm{C}$, then washed three times, and

a

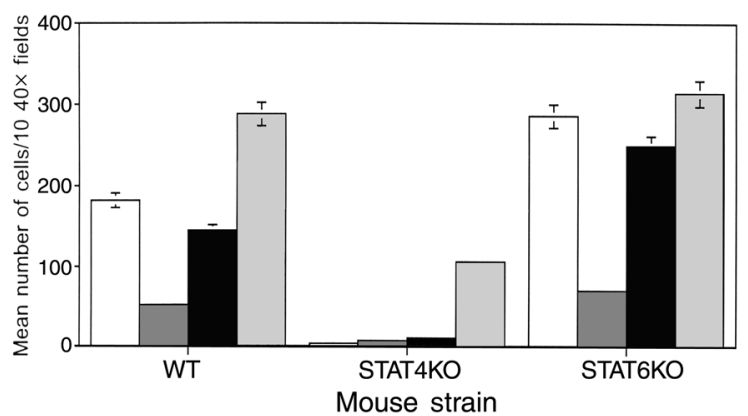

b

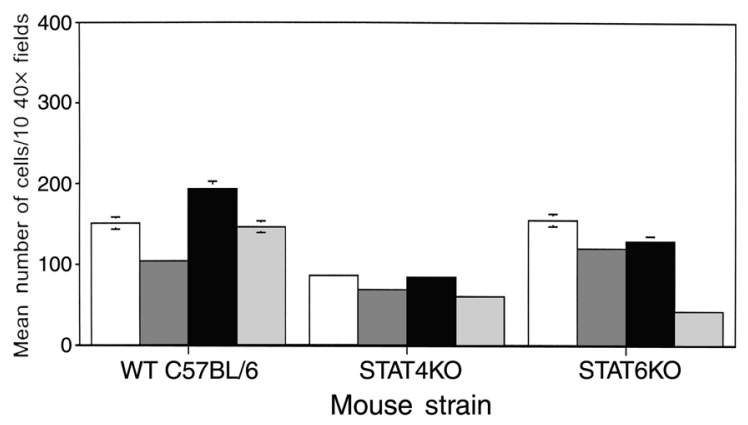




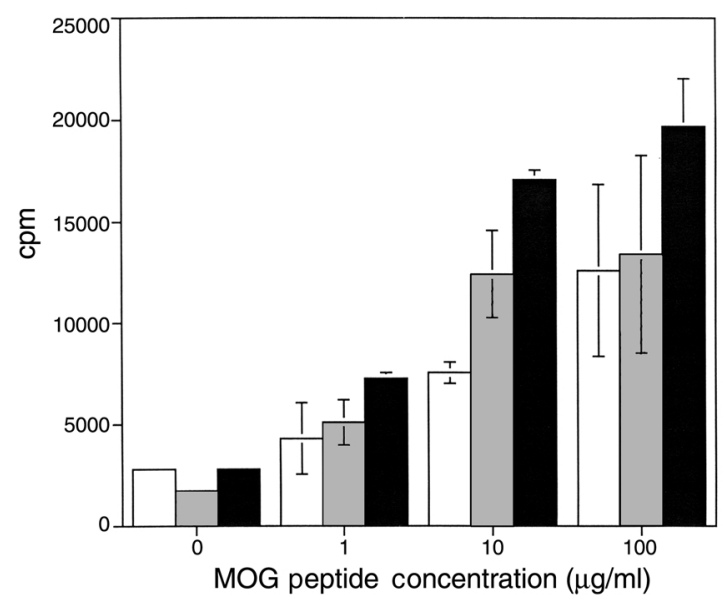

Figure 3

Proliferative response of splenocytes isolated from wild-type, STAT4 ${ }^{-/-}$, and STAT6-/- mice to MOG 35-55 in vitro. Splenocytes were obtained on day 14 postimmunization from wild-type (white bars), STAT4-/(gray bars), and STAT6-/- (black bars) mice. The cells were cultured in the presence of MOG 35-55 at 1, 10, and $100 \mu \mathrm{g} / \mathrm{ml}$ (on the $x$ axis). The cpm is indicated on the $y$ axis $( \pm$ SD). Proliferation to $10 \mu \mathrm{g} / \mathrm{ml}$ of MOG was significantly more in cultures from STAT4 $4^{-/}(P=0.0009)$ and STAT6 ${ }^{-1-}(P=0.0001)$ mice compared with wild-type.

horse anti-mouse IgG, IgG1, and IgG2a peroxidaseconjugated Abs (Vector Laboratories Inc.) were added (concentration 1:1,000 in PBS). Plates were incubated for 1 hour at room temperature. TMB Microwell Peroxidase Substrate (Kirkegaard \& Perry Laboratories, Gaithersburg, Maryland, USA) was used to develop the plates, and the reaction was stopped with TMB Stop Solution (Kirkegaard \& Perry Laboratories), and read at $450 \mathrm{~nm}$. Serum samples from immunized animals were compared to a naive serum standard, and the titer equivalent to the standard was recorded.

Statistical analysis. The Student's $t$ test was used to compare STAT4 -/- or STAT6 ${ }^{-/-}$groups to wild-type in the immunohistology, proliferation, and cytokine studies. The Mann-Whitney test was used to compare STAT4 ${ }^{-/}$and STAT6 $6^{-/}$groups to wild-type in the Ab study, while for the incidence comparisons, Fisher's exact test was used.

\section{Results}

Clinical disease expression in STAT4 $4^{--}$and STAT6 ${ }^{-/}$mice. First we tested the effects of targeted deletion of STAT4 versus STAT6 on the induction of EAE. Our data show that STAT4 $4^{-/-}$mice are resistant to the development of clinical EAE, while STAT6 ${ }^{-/}$mice experience a more severe form of disease as compared with STAT4 ${ }^{-/}$or wild-type mice (Figure 1). Composite data from three experiments are shown in Table 1. Mice were followed for up to 50 days. STAT4 $4^{--}$mice have a lower incidence of disease $(<50 \%)$ compared with wild-type and STAT $^{-/}$mice $(100 \% ; P=0.0008$ for either). The STAT4 $-/$ mice that develop EAE experience a similar day of onset of disease as wild-type mice, but show a very mild clinical course (mean maximal disease grade of $0.46 \pm 0.2)$. In contrast, STAT $6^{-/-}$mice have an earlier onset of disease compared with wild-type $(P<0.01)$ mice and attain a higher disease grade compared with wild-type $(P<0.005)$ and to STAT4 ${ }^{--}(P<0.0001)$ mice.

Immunopathology of the CNS after EAE induction. Pathologic examination of the CNS from STAT4-/, STAT $6^{-/}$, and wild-type mice taken on day 16 post immunization showed minimal infiltrates in the CNS of STAT4 $/-$ mice, while there were more infiltrates in the other two groups of mice (Figure 2a). Quantitation of $\mathrm{CD}^{+}$and $\mathrm{CD}^{+} \mathrm{T}$ cells, macrophages, and granulocytes after immunohistological staining showed significantly fewer infiltrating cells in STAT4 ${ }^{-/}$mice as compared with wild-type and STAT6 ${ }^{-/-}$mice (Figure 2a). There were significantly fewer $\mathrm{CD} 4^{+}$cells in spinal cord sections from STAT4 ${ }^{-/}$compared with wild-type mice taken on day 50 postimmunization (Figure 2b).

Cytokine production by primed T cells from STAT4-- and STAT6 ${ }^{-1-}$ mice. We examined the proliferation of in vivo primed splenocytes isolated from STAT4 $4^{--}$, STAT6 ${ }^{-/}$, and wild-type mice. T cells of STAT $4^{-/}$and STAT $6^{-/-}$mice proliferated well to MOG peptide in vitro (Figure 3), indicating that they are primed. We examined Th1 and Th2 cytokine production by ELISA assay and quantified the number of cytokine-secreting cells by ELISPOT assay. Interestingly, IFN- $\gamma$ production in culture supernatants was significantly higher in splenocytes of wild-type mice than in STAT6 ${ }^{-/}$mice after stimulation with MOG peptide (Figure 4a). This finding was confirmed by the
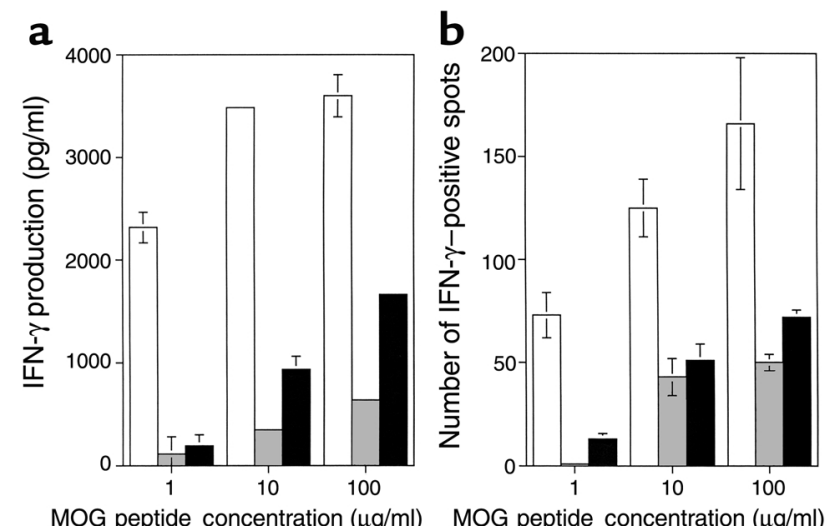

\section{Figure 4}

IFN- $\gamma$ production of splenocytes from wild-type, STAT4 ${ }^{-/}$, and STAT $6^{-/-}$mice in response to MOG peptide in vitro. (a) IFN- $\gamma$ production was measured by ELISA $( \pm S D)$ in the supernatants of splenocytes harvested on day 14 from C57BL/ 6 (white bars), STAT4 ${ }^{-/-}$(gray bars), or STAT6-/- mice (black bars) after 48 hours of culture with MOG 35 55 at concentrations of 1,10 , or $100 \mu \mathrm{g} / \mathrm{ml}$. IFN- $\gamma$ production was significantly greater in the cultures from wild-type mice compared with STAT4 ${ }^{-/-}$mice at all concentrations of MOG 35-55 $(P<0.005)$. (b) MOG 35-55-specific IFN- $\gamma$-producing cells $( \pm S D)$ were measured by ELISPOT assay in cultures of splenocytes from C57BL/ 6 wild-type (white bars), or STAT4 $4^{-/-}$(gray bars), or STAT6 ${ }^{-/-}$mice (black bars). The $y$ axis represents the number of positive cells per $2 \times 10^{5}$ cells plated. The frequency of IFN- $\gamma$-producing cells was significantly higher in wild-type than STAT4 ${ }^{-/-}(P<0.005)$ and STAT6 ${ }^{-1-}$ mice $(P<0.01)$ at all concentrations of MOG peptide. 

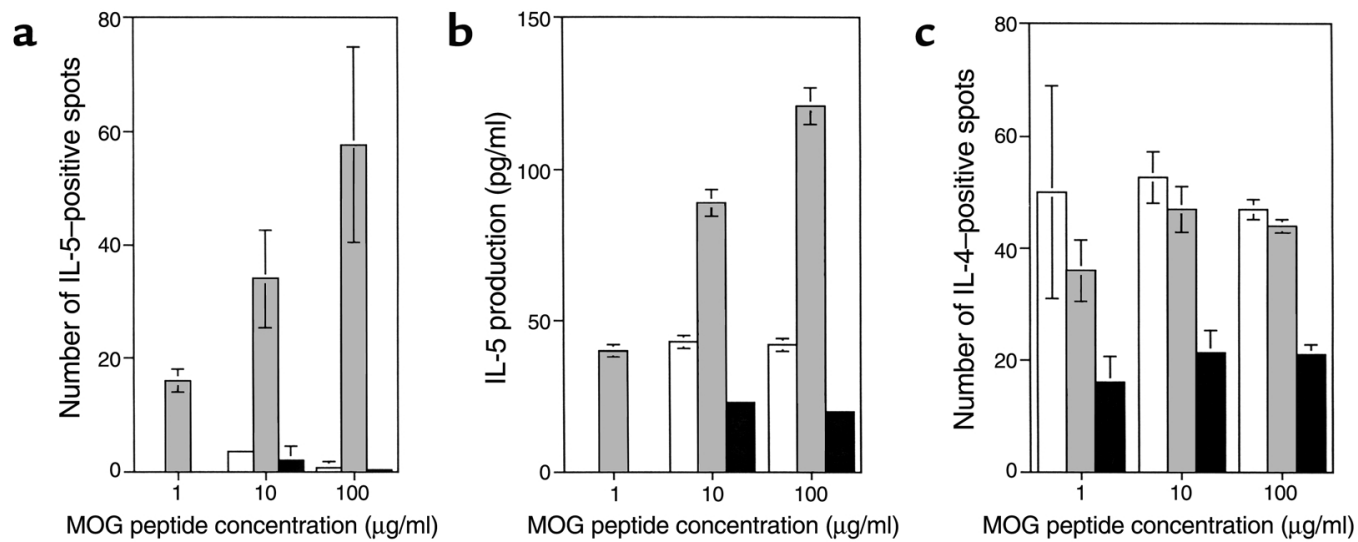

\section{Figure 5}

IL-4 and IL-5 cytokine production of splenocytes from wild-type, STAT4 ${ }^{-/-}$, and STAT6 ${ }^{-/-}$mice in response to MOG peptide in vitro. (a) IL-5 production was measured by ELISA $( \pm \mathrm{SD})$ in the supernatants of splenocytes harvested on day 14 postimmunization with MOG $\mathrm{p} 35-55$ from C57BL/ 6 wild-type (white bars), STAT4-/- (gray bars), or STAT6-/- mice (black bars), after 48 hours of culture with three different concentrations of MOG p35-55. Measurable IL-5 production was significantly greater in the cultures from STAT4 $/-$ mice at all concentrations of MOG p35-55 ( $P<0.05$ compared to wild-type and STAT6-/-). (b) MOG p35-55-specific IL-5-producing cells ( \pm SD) were measured by ELISPOT in cultures of splenocytes from C57BL/ 6 wild-type (white bars), STAT4-/- (gray bars), or STAT6 ${ }^{-/-}$mice (black bars) taken 14 days postimmunization with MOG p35-55. The $y$ axis represents the number of positive cells per $2 \times 10^{5}$ cells plated. The frequency of IL-5-producing cells was significantly higher in STAT4 ${ }^{-/-}$compared with wild-type or STAT6 ${ }^{-/-}$cultures at all concentrations of MOG $(P<0.005)$. (c) MOG p35-55-specific IL-4-producing cells ( \pm SD) were measured by ELISPOT in cultures of splenocytes from C57BL/6 wild-type (white bars), STAT4 ${ }^{-/-}$(gray bars), or STAT6 ${ }^{-/}$mice (black bars) taken from mice 14 days postimmunization with MOG p35-55. The $y$ axis represents the number of positive cells per $2 \times 10^{5}$ cells plated. The frequency of IL-4-producing cells was significantly higher in STAT4-/- and wildtype compared with STAT6 ${ }^{-1-}$ cultures at all concentrations of MOG $(P<0.01)$.

ELISPOT assay data showing the highest frequency of IFN- $\gamma$-producing cells in the wild-type cultures (Figure $4 \mathrm{~b})$. As expected, STAT4-/- mice had the lowest IFN- $\gamma$ production as determined by ELISA and the lowest frequency as determined by the ELISPOT assay (Figure 4, $a$ and b). IL-4 is difficult to measure in culture supernatants using ELISA due to consumption by cells; thus we measured IL-5 in culture supernatants as a marker of Th 2 cells. IL-5 production was highest in culture supernatants from STAT4 $^{-/-}$mice (Figure 5a). This was associated with a higher frequency of IL-5-producing cells as measured by ELISPOT assay in these mice (Figure $5 b$ ). The frequency of IL-4-producing cells as determined by ELISPOT assay was similar in the STAT4-/ and wild-type groups and significantly lower in the STAT6 ${ }^{-/-}$mice (Figure 5c).

Anti-MOG Ab titers in STAT4-/-, STAT6 ${ }^{-/}$, and wild-type mice. Anti-MOG Ab's were reported to augment disease severity in a MOG EAE rat model (25), although B cell-deficient C57BL/6 mice develop EAE (26). A decreased level of IgG1 and IgE, typically associated with a Th2 phenotype, have been reported in STAT6 $6^{-/}$ mice (16). IgG2a, typically associated with a Th1 phenotype, has been reported to be either elevated (27) or normal $(16,17)$ in STAT6-/- animals. We examined the relative titers of anti-MOG total IgG (Figure 6a), IgG1 (Figure 6b), and IgG2a (Figure 6c) from serum samples
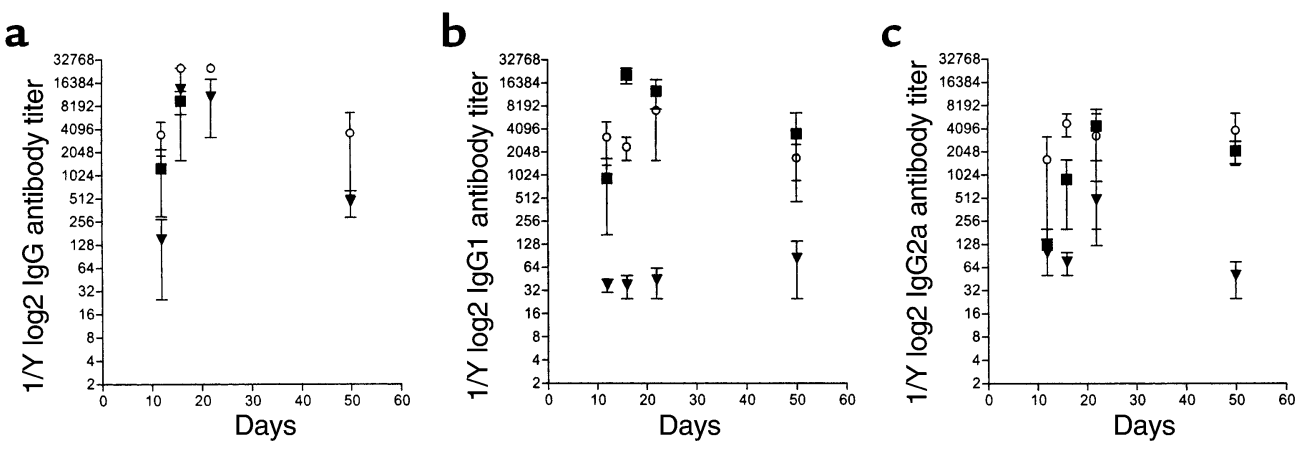

\section{Figure 6}

Anti-MOG Ab quantitation in STAT4 ${ }^{-/-}$, STAT6-/-, and wild-type mice. The relative titers of anti-MOG total IgG (a), $\operatorname{lgG} 1$ (b), and IgG2a (c) in serum samples from STAT4 ${ }^{-/-}$(open circles), STAT6 ${ }^{-/}$(filled triangles), and wild-type mice (filled squares) at four time points. Titer was determined by comparison to a naive serum sample. Serial 1:2 dilutions were made. The reciprocal of the titer (1/Y), at which the sample was the same as naive, was plotted on a $\log _{2}$ scale. Statistical analysis by the Mann-Whitney test shows no differences in total IgG titers among the three groups. IgG2a titers were not significantly different in either the STAT4-/- or STAT6-/- groups compared with wild-type mice. As anticipated, IgG1 titers were significantly lower in the STAT6 ${ }^{-/-}(P=0.028)$, but not STAT4 ${ }^{-/-}(P=$ NS $)$, mice compared with wild-type. 

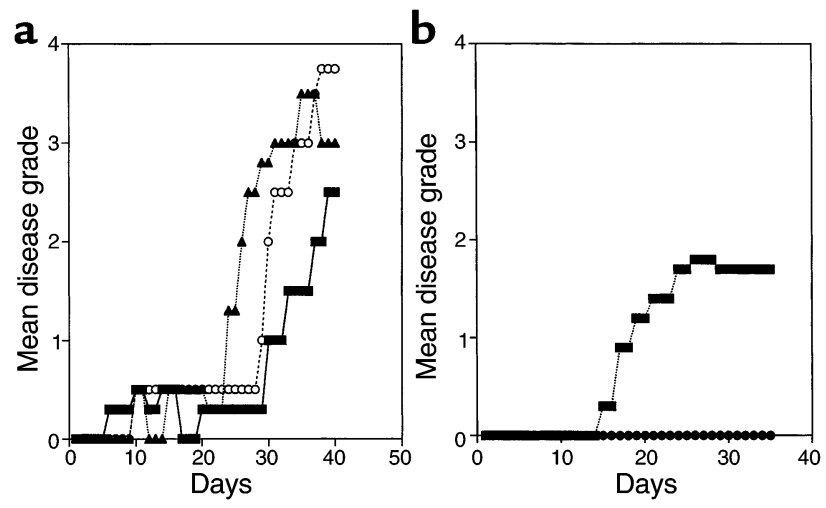

\section{Figure 7}

Adoptive transfer studies. (a) Adoptive transfer of wild-type cells into TCR- $\alpha \beta^{-/-}$mice. Splenocytes from wild-type mice were harvested 12 days postimmunization with MOG peptide. The cells were resuspended in PBS and wild-type splenocytes at concentrations of either $100 \times 10^{6}$ (open circles), $50 \times 10^{6}$ (filled triangles), or $25 \times 10^{6}$ (filled squares) were injected intraperitoneally into recipient TCR- $\alpha \beta^{-/-}$ mice. The recipients were immunized with MOG peptide 1 day after transfer. Five recipient mice per group were used. (b) Adoptive transfer of splenocytes from STAT4 $4^{-/-}$or STAT $6^{-/-}$mice into TCR- $\alpha \beta^{-/-}$ mice. Splenocytes from either wild-type STAT4 ${ }^{-/-}$or STAT6 $6^{-/-}$mice were harvested 12 days after immunization with MOG peptide. The cells were resuspended in PBS and $50 \times 10^{6}$ splenocytes from STAT4 $4^{-/}$mice (filled circles) or STAT6 ${ }^{-/-}$mice (filled squares) were injected intraperitoneally into recipient TCR- $\alpha \beta^{-/-}$mice. The recipients were immunized with MOG peptide 1 day after transfer. Five recipient mice per group were used.

isolated from STAT4 ${ }^{-/-}$, STAT6 $6^{-/}$, and wild-type mice at four different time points postimmunization: day $12,16,22$, and 50 . Three to five samples per group per time point were tested. Using Mann-Whitney analysis, no significant differences were found in total $\mathrm{IgG}$ titers between the three groups. IgG2a titers were not significantly different in either the STAT4 ${ }^{-/}$or STAT $6^{-/}$groups compared with wild-type mice. As anticipated, IgG1 titers were significantly lower in the STAT6 $^{-/-}(P=0.028)$ but not STAT4 ${ }^{-/-}(P=\mathrm{NS})$ mice compared with wild-type.

Adoptive transfer studies into TCR- $\alpha \beta^{-/-}$recipients. To further investigate the encephalitogenicity of Th1 and Th2 cells, we used an adoptive transfer model where primed splenocytes from mice immunized previously were transferred directly without in vitro stimulation into TCR- $\alpha \beta^{-/-}$animals. The TCR- $\alpha \beta^{-/-}$recipients were then immunized in order to activate the primed T cells in vivo. This method allows us to activate the donor cells in the cytokine environment being tested (see below), rather than activating the cells ex vivo and then transferring them into the recipient. Post immunization with MOG peptide, the recipients were observed for the development of clinical disease. TCR- $\alpha \beta^{-/-}$mice do not develop EAE postimmunization, but adoptive transfer of primed splenocytes from wild-type animals induced moderately severe EAE. The optimal number of wildtype cells for use in adoptive transfer was determined in dose-response experiments (Figure 7a) and $50 \times 10^{6}$ cells were found to be optimal (incidence, six of six; mean maximal grade, $3.5 \pm 0.7$; mean day of onset, $14.4 \pm 3.4$ ). Therefore, we used this adoptive transfer model system to study the putative encephalitogenic functions of Th1 versus Th 2 cells in vivo. When primed splenocytes from STAT $6^{-/-}$mice were adoptively transferred to TCR- $\alpha \beta^{-/-}$ recipients, they induced moderate disease (incidence, five of five; mean maximal grade, $1.8 \pm 1.2$; mean day of onset, $16.6 \pm 2.2$ ), while adoptive transfer of splenocytes from STAT4 $/-$ mice failed to cause EAE (incidence, zero of five; mean maximal grade, 0 ; mean day of onset, NA) (Figure $7 \mathrm{~b}$ ). These data confirm our active immunization studies showing that $\mathrm{T}$ cells from STAT $6^{-/-}$animals (Th1 biased) are encephalitogenic, while T cells from STAT4-/- (Th2 biased) are not.

Adoptive transfer of T cells into STAT4-/- recipients. To investigate the putative regulatory function of a Th2 environment on disease expression, we transferred $50 \times 10^{6}$ splenocytes from STAT6 ${ }^{-/}$or wild-type mice into STAT4 ${ }^{-/}$recipients that were subsequently immunized with MOG. Splenocytes harvested from STAT6 ${ }^{-/-}$mice resulted in worse disease than wild-type splenocytes when transferred into STAT4-/- recipients (Figure 8). This may be due to the ability of the Th2 environment to better regulate uncommitted wild-type T cells than STAT6 ${ }^{-/-}$cells, which lack the IL-4-regulated Th2 differentiation pathway and may only form Th1 cells. In vitro cytokine production confirmed this and showed high levels of IFN- $\gamma$ production in the supernatants of cultures of primed cells harvested from STAT4 ${ }^{-/}$recipients of STAT6 ${ }^{-/-}$ splenocytes (Figure 9a). IFN- $\gamma$ production in cultures of adoptively transferred wild-type cells were similar to STAT4 $4^{-/}$cell cultures with very little IFN- $\gamma$ production (Figure 9a). IL-5 levels were similar between STAT4 ${ }^{-/}$ recipients of STAT6 ${ }^{-/-}$cells or wild-type cells (Figure 9b).

\section{Discussion}

To our knowledge, this is the first report on the effect of targeted disruption of STAT 4 and STAT 6 genes on the development of EAE. The first novel observation is that STAT4 ${ }^{-/-}$mice are relatively protected from EAE, while STAT6 $6^{-/}$mice experience a more severe disease

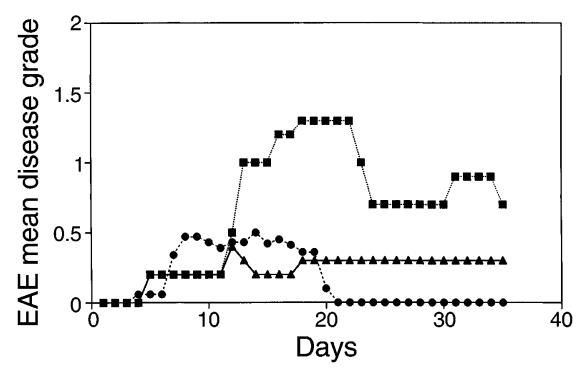

\section{Figure 8}

Adoptive transfer of wild-type splenocytes into a STAT4-/- host results in less disease than transfer of STAT $6^{-1-}$ splenocytes. Splenocytes $\left(50 \times 10^{6}\right)$ from wild-type (triangles) or STAT6 ${ }^{-/-}$(squares) mice were injected intraperitoneally into recipient STAT4 $/-$ mice that were then immunized as above. A nontransfer STAT4-/- group (circles) is also shown. Five recipient mice per group were used. 

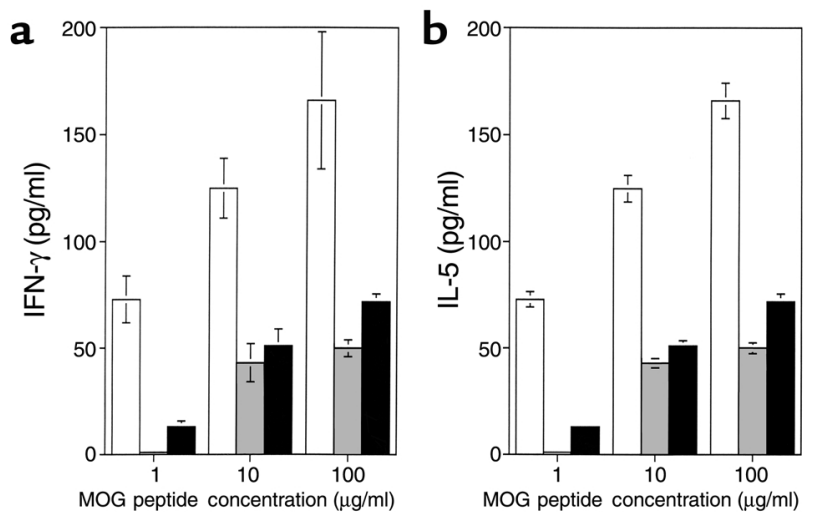

\section{Figure 9}

Cytokine production after adoptive transfer of T cells into STAT4 ${ }^{-/}$ recipients. Splenocytes were harvested on day 35 after immunization from STAT4 $4^{-/-}$and from STAT4 ${ }^{-/-}$recipients of wild-type and STAT6 ${ }^{-/-}$ cells. Splenocytes were cultured in vitro with various concentrations of MOG peptide. (a) IFN- $\gamma$ production ( \pm SD) was measured by ELISA in splenocytes harvested from control STAT4 ${ }^{--}$mice (white bars), STAT4 $/-$ recipients of either wild-type C57BL/ 6 splenocytes (gray bars), or STAT6 $6^{--}$splenocytes (black bars) on day 35 postimmunization. IFN- $\gamma$ production in STAT4 $4^{-/-}$recipients of STAT6 $6^{-/-}$splenocytes was greater than STAT4 ${ }^{-/}$recipients of wild-type splenocytes, which was similar to levels of IFN- $\gamma$ produced by STAT4 $4^{-/-}$mice immunized with MOG peptide with no transfer $(P<0.0001$ at MOG $10 \mu \mathrm{g} / \mathrm{ml})$. (b) IL-5 production $( \pm S D)$ was measured by ELISA in splenocytes harvested from control STAT4 ${ }^{-/-}$mice (white bars), STAT4-/- recipients of either wild-type C57BL/ 6 splenocytes (gray bars), or STAT6 ${ }^{-/}$splenocytes (black bars) on day 35 postimmunization. IL-5 production in STAT4-/recipients of wild-type splenocytes was greater than STAT4-/- recipients ofSTAT6 ${ }^{-/-}$splenocytes and STAT4 ${ }^{-/-}$mice with no transfer $(P<0.05$ at MOG $10 \mu \mathrm{g} / \mathrm{ml}$ and $P<0.001$ at MOG $100 \mu \mathrm{g} / \mathrm{ml}$ ).

than STAT4 $4^{-/-}$and wild-type controls. These data are different from those obtained with individual cytokine gene knockout mice and point to the potential therapeutic advantage of targeting genes that control Th1 and Th2 immune responses as opposed to individual cytokine genes. The Th1 cytokines, IFN- $\gamma$ and TNF- $\alpha$, have all been linked to disease expression in $\operatorname{EAE}(2,3$, $28,29)$. However, Th1 cytokines may play a dual role in disease initiation and disease remission $(10,30)$. The minimal clinical disease observed in STAT4 ${ }^{-/-}$mice is in sharp contrast to IFN- $\gamma^{-/}$mice that have massive CNS infiltrates and more severe disease than wild-type mice (ref. 9 and our own unpublished observations). This apparent paradox may be related to the regulatory functions of IFN- $\gamma$ as an anti-T cell proliferative cytokine (31-33) and, more relevant to our model, its potential role in inducing apoptosis of $\mathrm{T}$ cells in the CNS (34). STAT4 ${ }^{-/}$mice produce small amounts of IFN- $\gamma$ via a STAT4 independent pathway that may involve IL-18 (35-38). This small amount of IFN- $\gamma$ may be sufficient to regulate the immune response in STAT4-/- mice. Similarly, TNF- $\alpha$-deficient mice develop more severe $\operatorname{EAE}(39,40)$ than wild-type controls, again indicating that during some phase of the disease TNF- $\alpha$ plays a protective role. The role of IL- 12 in EAE is well documented: Ab's to IL-12 protect mice from passive disease (41) and from actively induced disease and relapses (42), while mice deficient in IL-12 are resistant to EAE (43). The functional effects of IL-12 are mediated via the IL-12R (44) after tyrosine phosphorylation of STAT4 $(45,46)$. Thus, our data showing that STAT4 ${ }^{-/-}$mice are protected from EAE are consistent with these observations.

There are several potential explanations for the lack of disease in STAT4 $/-$ mice. First, it is possible that cells in the STAT4 ${ }^{-/-}$mice fail to be primed. Our proliferation data show that STAT4-/--derived cells proliferate as well as the control cells, consistent with priming. Second, the cells may fail to differentiate and express the appropriate cytokine profile conducive to disease. Our data are consistent with this hypothesis, since cells from STAT4-/- mice produce low IFN- $\gamma$ and high IL-4 and IL-5. The third possibility is that STAT4 ${ }^{-/}$-primed cells lack the appropriate chemokine signals to enter the CNS. Th1 and Th2 cytokines play an important role in production of key chemokines that may control tissue migration of effector $\mathrm{T}$ cells $(47,48)$. Thus, the decrease in IFN- $\gamma$ in STAT4 $/-$ mice may decrease the production of Th1dependent chemokines such as IP-10 $(49,50)$ and MIG (51, 52), leading to milder disease. Using STAT4and STAT6-deficient mice, Zhang et al. recently reported a differential expression of chemokines by Th1 and Th 2 cells, and showed that the expression of Th2-derived chemokines was dependent on STAT6 and preferentially induced the chemotaxis of Th2 over Th1 cells (53). In an asthma model, Mathew et al. showed that antigen-specific wild-type Th2 cells adoptively transferred to STAT $6^{-/-}$mice fail to induce asthma consistent with there being functional STAT6 binding sites in the promoters of Th2 active chemokines (54). Furthermore, IFN- $\gamma$ plays a role in directing chemokine production so that IFN- $\gamma^{-/}$mice have undetectable levels of MCP-1 and RANTES during the course of EAE (30), although these chemokines are not decreased in STAT4 ${ }^{--}$mice (53). It appears that STAT proteins and other transcription factors such as NF- $\mathrm{KB}$ may bind to chemokine promoters (55) so that the STAT proteins may be a key point in the signaling pathway that leads to differentiation and recruitment of $\mathrm{T}$ cells.

It is interesting that STAT $6^{-/-}$mice have a more severe disease than wild-type mice despite having lower IFN- $\gamma$ production. Our data of the cytokine profiles in STAT4and STAT6-deficient mice suggest that the Th1/Th2 cytokine balance may be an important determinant of the expression pattern of clinical and pathological disease. Thus, in wild-type mice a higher amount of IFN- $\gamma$ production is counterbalanced by the production of Th2 cytokines, whereas in STAT6 $6^{-/}$mice a moderate production of IFN- $\gamma$ is unopposed by Th 2 cytokines leading to a more severe disease. STAT4 $/$ - mice, on the other hand, while producing a small amount of IFN- $\gamma$, express a predominance of Th 2 cytokines and are thus protected from disease. 
The second novel observation in our study is that primed STAT4 ${ }^{-/} \mathrm{T}$ cells do not induce disease in an immunodeficient host. The role of Th2 cells in EAE remains controversial. Th2 cells have been shown to protect against Th 1 cell-mediated inflammatory diseases $(56,57)$, and CNS-specific Th2 clones have been shown to be protective in $\operatorname{EAE}(58,59)$. However, there is conflicting data showing a lack of protection from disease by short-term proteolipid protein-specific Th2 cell lines (60). More importantly, myelin basic protein-specific Th2 cells induced delayed onset EAE in an immunodeficient host, associated with infiltration with polymorphonuclear leukocytes (61). Our data show a lack of encephalitogenicity of STAT4 ${ }^{-/}$cells in both immunocompetent and immunodeficient hosts. We have carried out our observations to day 50, and STAT4 ${ }^{-/}$remained resistant to clinical disease. In addition, in the STAT4 ${ }^{-/-}$ mice that developed very mild disease (44\%), the onset of disease was not delayed. Furthermore, STAT4 ${ }^{-/}$animals had minimal early and late cellular infiltrates including granulocytes. These observations emphasize the complexity of the Th1/Th2 paradigm in autoimmune diseases in general and in EAE in particular. We conclude that targeting STAT genes extends beyond the simple effect of a particular cytokine deficiency or dysregulation. Thus, our hypothesis is that the overall phenotype ( $T$ cell differentiation, recruitment, and chemotaxis) of the immune response determines the expression pattern of clinical and pathological disease in EAE.

The third novel and important observation in this study is that the Th2 environment in STAT4 ${ }^{-/}$animals may be regulatory, particularly in the case of adoptive transfer of primed wild-type splenocytes. Interestingly, primed STAT6 ${ }^{-/-}$cells mediated a more severe disease than wild-type cells in adoptive transfer experiments into STAT4 ${ }^{-/}$environment, raising the possibility that regulation is more effective when the target is less committed to develop into Th1 effector cells. Whether the regulatory environment in STAT4-/- animals is mediated by regulatory $\mathrm{T}$ cells or by a state of Th2 immune deviation with a predominance of Th2 cytokines and chemokines will require further investigation and possibly the generation of antigen-specific Th1 and Th2 lines and clones, as previously reported in an allogeneic system in vivo (62).

In summary, STAT4 and STAT6 genes control a pathway central to the induction of EAE. Disruption of the STAT4 gene ameliorates clinical disease and reduces inflammatory infiltrates in the CNS, while disruption of the STAT6 gene causes worse clinical and pathological disease. Our data show that modulation of T helper responses at a more proximal site, rather than complete elimination of a particular Th1 cytokine, is the key to modulating autoimmune responses in vivo. Further studies are required in relapsing models of STAT4- and STAT6-deficient mice in order to elucidate the mechanisms of function of these genes in EAE. We are currently backcrossing the STAT-deficient mice into the SJL background to study the effect of STAT4 and
STAT6 disruption on relapsing disease. Targeting individual cytokines have led to unwanted results in both humans and mice and care should be exercised in extrapolating these results to human diseases.

\section{Acknowledgments}

This work was supported by research grants from the National Multiple Sclerosis Society (RG-2589 to S.J. Khoury and FG 1287 to T. Chitnis) and the NIH (AI40945 to S.J. Khoury and PO1 AI-41521 to M.H. Sayegh). N. Najafian is a fellow of the National Kidney Foundation.

1. Powell, M.B., et al. 1990. Lymphotoxin and tumor necrosis factor-alpha production by myelin basic protein-specific $\mathrm{T}$ cell clones correlates with encephalitogenicity. Int. Immunol. 2:539-544.

2. Begolka, W.S., Vanderlugt, C.L., Rahbe, S.M., and Miller, S.D. 1998. Differential expression of inflammatory cytokines parallels progression of central nervous system pathology in two clinically distinct models of multiple sclerosis. J. Immunol. 161:4437-4446.

3. Issazadeh, S., Ljungdahl, A., Hojeberg, B., Mustafa, M., and Olsson, T. 1995. Cytokine production in the central nervous system of Lewis rats with experimental autoimmune encephalomyelitis: dynamics of mRNA expression for interleukin-10, interleukin-12, cytolysin, tumor necrosis factor alpha and tumor necrosis factor beta. J. Neuroimmunol. 61:205-212.

4. Issazadeh, S., Navikas, V., Schaub, M., Sayegh, M., and Khoury, S. 1998. Kinetics of expression of costimulatory molecules and their ligands in murine relapsing experimental autoimmune encephalomyelitis in vivo. J. Immunol. 161:1104-1112.

5. Khoury, S.J., Hancock, W.W., and Weiner, H.L. 1992. Oral tolerance to myelin basic protein and natural recovery from experimental autoimmune encephalomyelitis are associated with downregulation of inflammatory cytokines and differential upregulation of transforming growth factor beta, interleukin 4, and prostaglandin E expression in the brain. J. Exp. Med. 176:1355-1364.

6. Liblau, R., Steinman, L., and Brocke, S. 1997. Experimental autoimmune encephalomyelitis in IL-4-deficient mice. Int. Immunol. 9:799-803.

7. Bettelli, E., et al. 1998. IL-10 is critical in the regulation of autoimmune encephalomyelitis as demonstrated by studies of IL-10- and IL-4-deficient and transgenic mice. J. Immunol. 161:3299-3306.

8. Falcone, M., Rajan, A.J., Bloom, B.R., and Brosnan, C.F. 1998. A critical role for IL-4 in regulating disease severity in experimental allergic encephalomyelitis as demonstrated in IL-4-deficient C57BL/ 6 mice and BALB/c mice. J. Immunol. 160:4822-4830.

9. Ferber, I.A., et al. 1996. Mice with a disrupted IFN-gamma gene are susceptible to the induction of experimental autoimmune encephalomyelitis (EAE). J. Immunol. 156:5-7.

10. Willenborg, D.O., Fordham, S., Bernard, C.C., Cowden, W.B., and Ramshaw, I.A. 1996. IFN-gamma plays a critical down-regulatory role in the induction and effector phase of myelin oligodendrocyte glycoprotein-induced autoimmune encephalomyelitis. J. Immunol. 157:3223-3227.

11. Duong, T.T., St. Louis, J., Gilbert, J.J., Finkelman, F.D., and Strejan, G.H. 1992. Effect of anti-interferon-gamma and anti-interleukin-2 monoclonal antibody treatment on the development of actively and passively induced experimental allergic encephalomyelitis in the SJL/J mouse. J. Neuroimmunol. 36:105-115.

12. Lublin, F.D., et al. 1993. Monoclonal anti-gamma interferon antibodies enhance experimental allergic encephalomyelitis. Autoimmunity. 16:267-274.

13. Lin, J.X., et al. 1995. The role of shared receptor motifs and common Stat proteins in the generation of cytokine pleiotropy and redundancy by IL2, IL-4, IL-7, IL-13, and IL-15. Immunity. 2:331-339.

14. Karnitz, L.M., and Abraham, R.T. 1995. Cytokine receptor signaling mechanisms. Curr. Opin. Immunol. 7:320-326.

15. Onishi, M., Nosaka, T., and Kitamura, T. 1998. Cytokine receptors: structures and signal transduction. Int. Rev. Immunol. 16:617-634.

16. Takeda, K., et al. 1996. Essential role of Stat6 in IL-4 signalling. Nature. 380:627-630.

17. Kaplan, M.H., Schindler, U., Smiley, S.T., and Grusby, M.J. 1996. Stat6 is required for mediating responses to IL- 4 and for development of Th2 cells. Immunity. 4:313-319.

18. Jacobson, N.G., et al. 1995. Interleukin 12 signaling in T helper type 1 (Th1) cells involves tyrosine phosphorylation of signal transducer and activator of transcription (Stat)3 and Stat4. J. Exp. Med. 181:1755-1762.

19. Thierfelder, W.E., et al. 1996. Requirement for Stat4 in interleukin-12mediated responses of natural killer and T cells. Nature. 382:171-174. 
20. Kaplan, M.H., Sun, Y.L., Hoey, T., and Grusby, M.J. 1996. Impaired IL-12 responses and enhanced development of Th2 cells in Stat4-deficient mice. Nature. 382:174-177.

21. Kaplan, M.H., Wurster, A.L., and Grusby, M.J. 1998. A signal transducer and activator of transcription (Stat)4-independent pathway for the development of T helper type 1 cells. J. Exp. Med. 188:1191-1196.

22. Holz, A., et al. 1999. Disruption of the STAT4 signaling pathway protects from autoimmune diabetes while retaining antiviral immune competence. J. Immunol. 163:5374-5382.

23. Kishimoto, K., et al. 2000. The role of CD154-CD40 versus CD28-B7 costimulatory pathways in regulating allogeneic Th1 and Th2 responses in vivo. J. Clin. Invest. 106:63-72.

24. Chitnis, T., et al. 2001. CD28-independent induction of experimental autoimmune encephalomyelitis. J. Clin. Invest. 107:575-583.

25. Linington, C., Bradl, M., Lassmann, H., Brunner, C., and Vass, K. 1988. Augmentation of demyelination in rat acute allergic encephalomyelitis by circulating mouse monoclonal antibodies directed against a myelin/oligodendrocyte glycoprotein. Am. J. Pathol. 130:443-454.

26. Hjelmstrom, P., Juedes, A.E., Fjell, J., and Ruddle, N.H. 1998. B-cell-deficient mice develop experimental allergic encephalomyelitis with demyelination after myelin oligodendrocyte glycoprotein sensitization. $J$. Immunol. 161:4480-4483.

27. Shimoda, K., et al. 1996. Lack of IL-4-induced Th2 response and IgE class switching in mice with disrupted Stat6 gene. Nature. 380:630-633.

28. Issazadeh, S., et al. 1996. Cytokines in relapsing experimental autoimmune encephalomyelitis in DA rats: persistent mRNA expression of proinflammatory cytokines and absent expression of interleukin-10 and transforming growth factor-beta. J. Neuroimmunol. 69:103-115.

29. Issazadeh, S., et al. 1995. Interferon gamma, interleukin 4 and transforming growth factor beta in experimental autoimmune encephalomyelitis in Lewis rats: dynamics of cellular mRNA expression in the central nervous system and lymphoid cells. J. Neurosci. Res. 40:579-590.

30. Tran, E.H., Prince, E.N., and Owens, T. 2000. IFN-gamma shapes immune invasion of the central nervous system via regulation of chemokines. J. Immunol. 164:2759-2768.

31. Konieczny, B.T., et al. 1998. IFN-gamma is critical for long-term allograft survival induced by blocking the CD28 and CD40 ligand T cell costimulation pathways. J. Immunol. 160:2059-2064.

32. Hassan, A.T., et al. 1999. Regulation of alloantigen-mediated T-cell proliferation by endogenous interferon-gamma: implications for long-term allograft acceptance. Transplantation. 68:124-129.

33. Badovinac, V.P., Tvinnereim, A.R., and Harty, J.T. 2000. Regulation of antigen-specific CD8+ T cell homeostasis by perforin and interferongamma. Science. 290:1354-1358.

34. Chu, C.Q., Wittmer, S., and Dalton, D.K. 2000. Failure to suppress the expansion of the activated CD4 $\mathrm{T}$ cell population in interferon gammadeficient mice leads to exacerbation of experimental autoimmune encephalomyelitis. J. Exp. Med. 192:123-128.

35. Barbulescu, K., et al. 1998. IL-12 and IL-18 differentially regulate the transcriptional activity of the human IFN-gamma promoter in primary CD4+ T lymphocytes. J. Immunol. 160:3642-3647.

36. Robinson, D., et al. 1997. IGIF does not drive Th1 development but synergizes with IL-12 for interferon-gamma production and activates IRAK and NFkappaB. Immunity. 7:571-581.

37. Xu, D., et al. 1998. Selective expression and functions of interleukin 18 receptor on $\mathrm{T}$ helper (Th) type 1 but not Th2 cells. J. Exp. Med. 188:1485-1492.

38. Yoshimoto, T., et al. 1998. IL-12 up-regulates IL-18 receptor expression on T cells, Th1 cells, and B cells: synergism with IL-18 for IFN-gamma production. J. Immunol. 161:3400-3407.

39. Frei, K., et al. 1997. Tumor necrosis factor alpha and lymphotoxin alpha are not required for induction of acute experimental autoimmune encephalomyelitis. J. Exp. Med. 185:2177-2182.

40. Liu, J., et al. 1998. TNF is a potent anti-inflammatory cytokine in autoimmune-mediated demyelination. Nat. Med. 4:78-83.

41. Leonard, J.P., Waldburger, K.E., and Goldman, S.J. 1995. Prevention of experimental autoimmune encephalomyelitis by antibodies against interleukin 12. J. Exp. Med. 181:381-386.

42. Constantinescu, C.S., et al. 1998. Antibodies against IL-12 prevent superantigen-induced and spontaneous relapses of experimental autoimmune encephalomyelitis. J. Immunol. 161:5097-5104.

43. Segal, B.M., Dwyer, B.K., and Shevach, E.M. 1998. An interleukin (IL)10/IL-12 immunoregulatory circuit controls susceptibility to autoimmune disease. J. Exp. Med. 187:537-546.

44. Chang, J.T., Shevach, E.M., and Segal, B.M. 1999. Regulation of interleukin (IL)-12 receptor beta2 subunit expression by endogenous IL-12: a critical step in the differentiation of pathogenic autoreactive T cells. $J$. Exp. Med. 189:969-978.

45. Jacobson, N.G., et al. 1995. Interleukin 12 signaling in T helper type 1 (Th1) cells involves tyrosine phosphorylation of signal transducer and activator of transcription (Stat)3 and Stat4. J. Exp. Med. 181:1755-1762.

46. Bacon, C.M., et al. 1995. Interleukin 12 induces tyrosine phosphorylation and activation of STAT4 in human lymphocytes. Proc. Natl. Acad. Sci. USA. 92:7307-7311.

47. Sallusto, F., Mackay, C.R., and Lanzavecchia, A. 2000. The role of chemokine receptors in primary, effector, and memory immune responses. Annu. Rev. Immunol. 18:593-620.

48. Luster, A.D. 1998. Chemokines-chemotactic cytokines that mediate inflammation. N. Engl. J. Med. 338:436-445.

49. Luster, A.D., and Ravetch, J.V. 1987. Biochemical characterization of a gamma interferon-inducible cytokine (IP-10). J. Exp. Med. 166:1084-1097.

50. Kaplan, G., Luster, A.D., Hancock, G., and Cohn, Z.A. 1987. The expression of a gamma interferon-induced protein (IP-10) in delayed immune responses in human skin. J. Exp. Med. 166:1098-1108.

51. Farber, J.M. 1997. Mig and IP-10: CXC chemokines that target lymphocytes. J. Lenkoc. Biol. 61:246-257.

52. Liao, F., et al. 1995. Human Mig chemokine: biochemical and functional characterization. J. Exp. Med. 182:1301-1314.

53. Zhang, S., Lukacs, N.W., Lawless, V.A., Kunkel, S.L., and Kaplan, M.H. 2000. Cutting edge: differential expression of chemokines in Th1 and Th2 cells is dependent on Stat6 but not Stat4. J. Immunol. 165:10-14.

54. Mathew, A., et al. 2001. Signal transducer and activator of transcription 6 controls chemokine production and thelper cell type 2 cell trafficking in allergic pulmonary inflammation. J. Exp. Med. 193:1087-1096.

55. Matsukura, S., et al. 1999. Activation of eotaxin gene transcription by NF-kappa B and STAT6 in human airway epithelial cells. J. Immunol. 163:6876-6883

56. Rocken, M., Racke, M., and Shevach, E.M. 1996. IL-4-induced immune deviation as antigen-specific therapy for inflammatory autoimmune disease. Immunol. Today. 17:225-231.

57. Liblau, R.S., Singer, S.M., and McDevitt, H.O. 1995. Th1 and Th2 CD4+ $\mathrm{T}$ cells in the pathogenesis of organ-specific autoimmune diseases. Immunol. Today. 16:34-38.

58. Kuchroo, V.K., et al. 1995. B7-1 and B7-2 costimulatory molecules activate differentially the Th1/Th2 developmental pathways: application to autoimmune disease therapy. Cell. 80:707-718.

59. Chen, Y., Kuchroo, V.K., Inobe, J., Hafler, D.A., and Weiner, H.L. 1994. Regulatory $\mathrm{T}$ cell clones induced by oral tolerance: suppression of autoimmune encephalomyelitis. Science. 265:1237-1240.

60. Khoruts, A., Miller, S.D., and Jenkins, M.K. 1995. Neuroantigen-specific Th2 cells are inefficient suppressors of experimental autoimmune encephalomyelitis induced by effector Th1 cells. J. Immunol. 155:5011-5017.

61. Lafaille, J.J., et al. 1997. Myelin basic protein-specific T helper 2 (Th2) cells cause experimental autoimmune encephalomyelitis in immunodeficient hosts rather than protect them from the disease. J. Exp. Med. 186:307-312

62. Waaga, A.M., et al. 2001. Regulatory functions of self-restricted MHC class II allopeptide-specific Th2 clones in vivo. J. Clin. Invest. 107:909-916. 